\title{
A Fatal Case of Chronic Active EBV Infection
}

\author{
Mehta $D^{1 *}$, Deplano S ${ }^{1}$, Olavarria $E^{1}$, Gaur $P^{2}$ and \\ Bourantas $\mathbf{L}^{1}$ \\ ${ }^{1}$ Department of Haematology, Imperial College NHS \\ Trust, UK \\ ${ }^{2}$ Department of Radiology, Imperial College NHS Trust, \\ UK \\ *Correspondling author: Mehta D, Department of \\ Haematology, Hammersmith Hospital, Imperial College \\ NHS Trust, London, UK
}

Received: November 30, 2020; Accepted: January 05, 2021; Published: J anuary 12, 2021

\section{Abstract}

Chronic active EBV infection is a rare condition with considerable diagnostic and treatment difficulties. We herein report the case of a 27 -year old female presenting with headaches and diplopia who deteriorated rapidly following her diagnosis, with confirmed EBV-infected T-lymphocyte deposits in multiple tissue sites including temporalis muscle and liver.

Keywords: Ebstein-Barr virus; T-lymphocytes; Allogenic bone marrow transplant; Chronic active EBV infection

\section{Introduction}

Chronic Active Epstein-Barr Virus Infection (CAEBV) is a rare disease caused by the persistent presence of EBV predominantly in T-lymphocytes [1]. Symptoms include persistent fever, hepatosplenomegaly, hepatitis and lymphadenopathy following Infectious Mononucleosis (IM). Skin rashes and pancytopenia are also common. Complications, which can be life-threatening, occur as a result of T-lymphocyte infiltration of tissues. Such complications include haemophagocytic lymphohistiocytosis, liver failure, coronary artery aneurysms, and T-cell lymphomas. The following are the current diagnostic criteria: (I) IM-like symptoms persisting more than 3 months; (II) increased EBV DNA (>102.5 copies/mg) in peripheral blood; (III) histological evidence of organ disease; (IV) demonstration of EBV RNA or viral protein in affected tissues [2]. A diagnosis should be made in patients without known immunodeficiency. Pathogenesis of CAEBV remains unclear. Whilst CAEBV by definition occurs in immunocompetent patients, it is possible that those patients affected have aberrant activity of EBV-specific cytotoxic T-cells. There also appear to be well-defined racial differences in the susceptibility to CAEBV, therefore suggesting a genetic component. The majority of reported cases have arisen from East Asia. There is currently no well-established treatment approach. Haematopoietic Stem-Cell Transplantation (HSCT) remains the only curative treatment, without which prognosis is poor [3]. Immunosuppressive therapies including corticosteroids, ciclosporin and azathioprine have been trialled, although with only transient clinical improvement demonstrated [4]. The use of antiviral drugs such as ganciclovir and valganciclovir has lacked evidence of efficacy. By reducing the burden of residual EBVinfected T-cells, chemotherapy is another consideration. However, there remains considerable uncertainty as to the appropriate timing of chemotherapy.

\section{Case Presentation}

A 27-year old female was referred to the haematology clinic with splenomegaly, thrombocytopenia and leukopenia. There were no B symptoms. She reported symptoms of infectious mononucleosis 6 months previously and had also recently developed migraine-like headaches. She had no significant past medical history and was not taking any regular medications. She was a non-smoker and drank approximately 14 units of alcohol per week. There was no relevant family history. Examination revealed palpable splenomegaly with no lymphadenopathy. Initial blood tests showed $\mathrm{Hb} 123 \mathrm{~g} / \mathrm{L}$, WBC 2.8x109/L, platelets 120x109/L, neutrophils $1.7 \times 109 / \mathrm{L}$. Iron studies showed iron deficiency, however vitamin B12 and folate levels were normal. Renal and liver function were also normal. Epstein-Barr nuclear antigen-1 (EBNA-1) IgG was detected, consistent with past EBV infection. HIV and hepatitis B/C tests were negative. An abdominal ultrasound scan showed splenomegaly with a spleen length of $16.8 \mathrm{~cm}$. Over the next few months, the patient continued to suffer from headaches and developed a new left partial $\mathrm{VI}^{\text {th }}$ nerve palsy and a papular rash over her face and torso. An MRI brain scan showed orbital inflammation, suggesting a possible systemic inflammatory condition. CSF examination revealed no abnormal cells and was negative for viruses including EBV. Skin biopsy was normal. Progressively worsening splenomegaly was demonstrated on repeat imaging, with a spleen length of $18.3 \mathrm{~cm}$. A bone marrow aspirate and trephine biopsy showed hypercellular marrow with no evidence of lymphomatous involvement, and no abnormal lymphocyte population identified on flow cytometry. Further blood tests showed mildly positive anti-neutrophil antibodies, indicating autoimmune neutropenia, as well as clonality on T-Cell Receptor (TCR) gene rearrangement. A whole-body PET/CT scan showed splenomegaly but no evidence of underlying malignancy. A subsequent deterioration in liver function, with alkaline phosphatase rising to $945 \mathrm{unit} / \mathrm{L}$ and alanine transferase rising to $192 \mathrm{unit} / \mathrm{L}$, led to a transjugular liver biopsy. In situ hybridisation with the EBV-encoded small RNA (EBER-ISH) demonstrated positivity in a large number of cells, suggestive of an EBV-driven T-cell cytotoxic reaction. There was also a notably increasing EBV viraemia, rising from 100,000 copies/ml to 1.4 million copies/ml over a 2-week period. A further bone marrow biopsy showed no demonstrable evidence of lymphoma but did show EBER-ISH-positive lymphoid cells totalling 1-2 \%. A peripheral blood EBV cell-subset test showed the presence of EBV in T-lymphocytes and NK cells, but not in B-lymphocytes. Temporalis muscle MRI imaging was performed after the patient developed profound peri-orbital oedema and worsening of diplopia. This showed significant oedema and diffuse high T2 signal in the body of the right temporalis muscle. EBER-ISH on temporalis muscle biopsy showed substantial presence of EBV-positive lymphoid cells. With a 


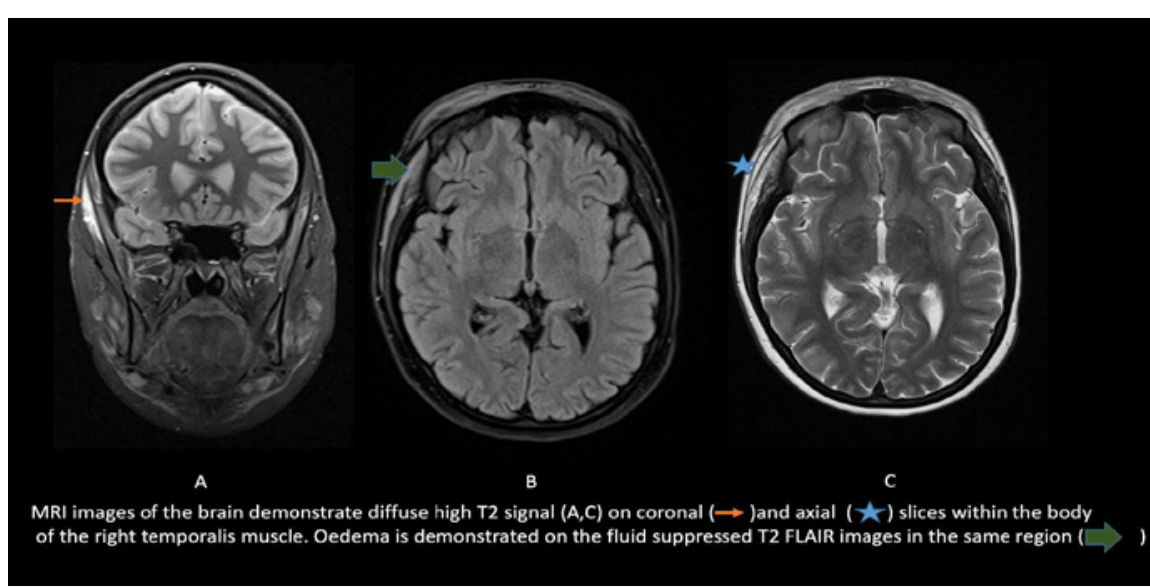

Figure 1:

diagnosis of T-cell CAEBV involving multiple tissue sites, further tests were conducted to rule out primary immunodeficiency. Perforin and cytokine responses, and soluble CD25 levels, were normal. Genetic screening of the immunodeficiency gene panel by next generation sequencing identified heterozygosity for FASLG and PRKDC mutations. The significance of these results was unclear, however FAS-mediated apoptosis is a well-described aspect of lymphocyte homeostasis, and defects in this pathway impair programmed cell death of lymphocytes [5]. Autoimmune Lymphoproliferative Syndrome (ALPS) is a condition which presents in early childhood with chronic lymphadenopathy and auto-immunity, and is associated with mutations in FAS, FAS-L and other downstream molecules in the lymphocyte apoptosis-inducing complex. Other conditions such as SLE have also been associated with such FAS polymorphisms. The patient received short courses of IV methylprednisolone following flares of diplopia, which led to dramatic though temporary clinical improvement. Oral valganciclovir was also commenced, as well as monthly doses of intravenous immunoglobulin at $1 \mathrm{~g} / \mathrm{kg}$. Despite these measures, the eye symptoms worsened, and the patient developed fevers, worsening hepatitis and new-onset thrombocytopenia. An EBV-specific cytotoxic T-cell infusion obtained from the father was administered without success. Definitive curative treatment would only have been possible with allogeneic HSCT, however the patient unfortunately continued to deteriorate and eventually developed multi-organ failure leading to death.

\section{Discussion}

CAEBV is a rare disease in the Western world, which is difficult to diagnose. When tissue samples are obtained, microscopic findings show predominantly non-specific inflammatory changes, and as a result reaching a pathological diagnosis is challenging. The key is in the detection of EBV in infiltrated lymphocytes using EBERISH. Whilst immunosuppressive therapy can yield transient clinical improvement, curative treatment can only be achieved by allogeneic HSCT. Without this, prognosis is extremely poor and death can be rapid (Figure 1).

\section{References}

1. Kimura H, Ito $\mathrm{Y}$, Kawabe S, Gotoh K, Takahashi Y, Kojima S, et al. EBV-associated T/NK-cell lymphoproliferative diseases in nonimmunocompromised hosts: prospective analysis of 108 cases. Blood. 2012; 119: 673-686.

2. Quintanilla-Martinez L, Ko Y-H, Kimura H, Jaffe ES. EBV-positive T-cell and NK-cell lymphoproliferative diseases of childhood. In: Swerdlow SH, Campo $E$, Harris NL, Jaffe ES, Pileri SA, Stein $\mathrm{H}$, et al, editors. Who Classification of Tumours of Haematopoietic and Lymphoid Tissues. Lyon, France: IARC. 2017; 355-363.

3. Bollard CM, Cohen Jl. How I treat T-cell chronic active Epstein-Barr virus disease. Blood. 2018; 131: 2899-2905.

4. Cohen JI, Jaffe ES, Dale JK, Pittaluga S, Heslop H, Rooney C, et al. Characterization and treatment of chronic active Epstein-Barr virus disease: a 28-year experience in the United States. Blood. 2011; 117: 5835-5849.

5. Worth A, Thrasher AJ, Gaspar HB. Autoimmune lymphoproliferative syndrome: molecular basis of disease and clinical phenotype. $\mathrm{Br} \mathrm{J}$ Haematol. 2006; 133: 124-140. 